\title{
Little Artifices of Huge Libraries: Secrets of Parallel Liquid-Phase Synthesis
}

\author{
E. V. Babaev \\ Lomonosov Moscow State University, Vorob'evy Gory 1, Moscow, 119991 Russia \\ e-mailbabaev@org.chem.msu.ru \\ Received July 10, 2009
}

\begin{abstract}
Key aspects of differences between liquid-phase parallel synthesis and preparation of a single compound at a time are reviewed. Specific technique and equipment is required to make in the parallel manner such processes as heating (involving MW ovens and flow microreactors), extraction, filtration, and chromatography. Rich illustrations may be useful in educational courses on medicinal and combinatorial chemistry.
\end{abstract}

DOI: $10.1134 / \mathrm{S} 1070363210120273$

In the previous papers we considered the possibilities of solid-phase synthesis (SPS) of libraries of chemical compounds, and, therewith, some of these methods $[1,2]$ can be used as training tasks at student laboratories. All manipulations in SPS are in fact limited to two multiply repeated operations: addition of an outside reagent (reaction as such) and its removal (filtering and washing). The technology may slightly vary, but a common feature of all variants is that a reactor is combined with a filter for purification. In this sense, the difference between a tube with a filter (billboard) and a porous plastic container ("tea bag") is not too big. A lantern combines both these principle, and filtration comes down to routine washing of a macroscopic object.

The advantages of SPS over usual liquid-phase synthesis (LPS) are undeniable: access to large combinatorial libraries with moderate expenses, possibility of automation of the process, higher yields of the target product due to a large excess of reagents, and facility of purification, associated with the fact that excess reagents, by-products, and salts are readily removed at the washing stage. The method is ideally suited in cases when one has, at different stages, to combine or isolate intermediate compounds, involving them in complicated reaction sequences. The SPS technology allows successful labeling of multiply changed objects. This relates to so-called tagging technologies [2].

If conditions are thoroughly selected and optimized (this may take 1-3 months; Fig. 1), SPS can provide more than 500 compounds per month. However, the SPS methodology has certain limitations.

First, only a relatively small fraction of reactions is adapted to occur on supports. It is hard to imagine that many traditional reactions (sulfonation, nitration, and even bromination) with a molecule grafted on a polystyrene support; it should also not be forgotten that linkers are sensitive to acids and alkalis. Second, introduction of "new" reactions into SPS takes a long time, since the entire synthetic sequence (including separation of support and product) should be perfectly

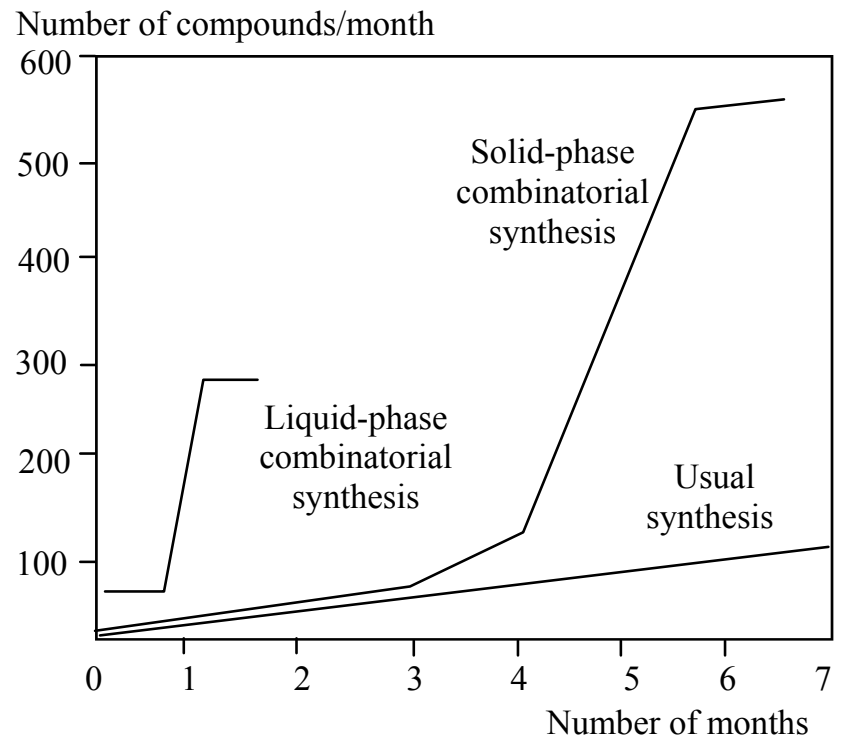

Fig. 1. Productivity of different methods of synthesis 
(a)

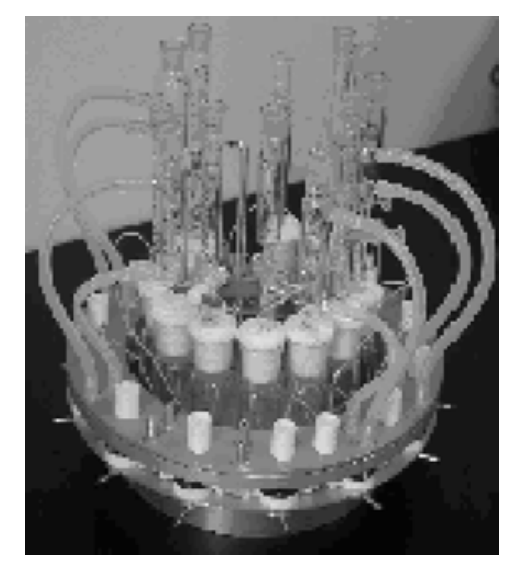

(b)

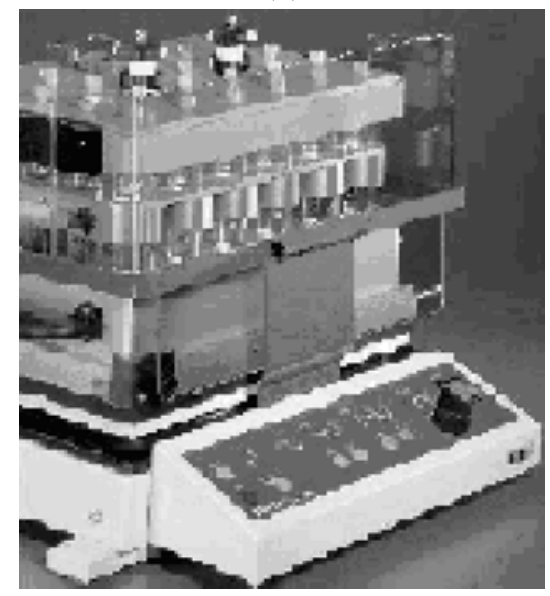

Fig. 2. Parallel refluxing of a great number of reaction vessels in (a) CombiSyn and (b) SynCore.

worked out. Third, solid reagents and insoluble catalysts cannot be applied in SPS. Fourth, SPS is accomplished almost "blindly," since intermediate products are difficult to identify. Finally, many popular supports and linkers for SPS are expensive.

Thus, to accomplish parallel reaction in a usual LPS variant (the commonly accepted term is wet chemistry) is frequently more reasonable and sometimes unavoidable. Therewith, it takes less time to optimize LPS compared to SPS (Fig. 1). In practice both methodologies complement each other. Development of parallel LPS technologies already has its own history (see the review [3]), and some most illustrative details are discussed in the present review.

(a)
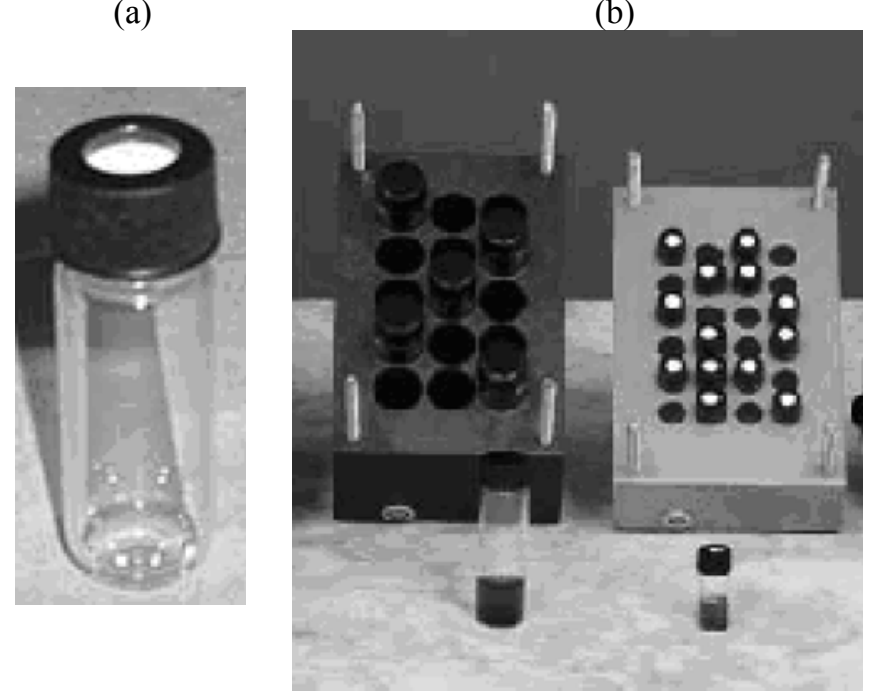

Fig. 3. Vials for parallel synthesis, a substitute of a reflux condenser.

\section{Technology of Parallel Liquid-Phase Synthesis}

The essential attributes of classical LPS are multinecked flasks with reflux condensers, dropping funnels, and thermometers, which are usually heated on magnetic stirrers. Obviously, to perform tens and hundreds reactions in parallel, an alternative to this conventional laboratory equipment is required. In view of our long-term experience in training students in the framework of the special laboratory course in combinatorial chemistry at the MSU, we will consider here both the most modern expensive equipment and routine facilities (the classical principle of "sealing wax and rope") for a low-budget laboratory.

\section{Parallel Refluxing}

A great number of patented solutions as to how to combine several reaction vessels into a single construction are available. A smart solution is our tested apparatus CombiSyn (Fig. 2a). The apparatus was designed by M. Baru (Pushchino, Moscow Region, Russia) and was previously distributed via Chemical Diversity. As seen, ordinary reflux condensers have a common circulation system, which allows a compact arrangement of ordinary reaction vessels. The apparatus functions by the carousel principle, and up to 13 reaction vessels can be refluxed and stirred by means one a single magnetic stirrer.

The second model we tested is a Buchi parallel synthesis apparatus SynCore (Fig. 2b). This model is envisioned to accommodate 24 reaction vessels. Heating and stirring are performed by means of a temperature-controlled shaker. The SynCore apparatus 
(a)

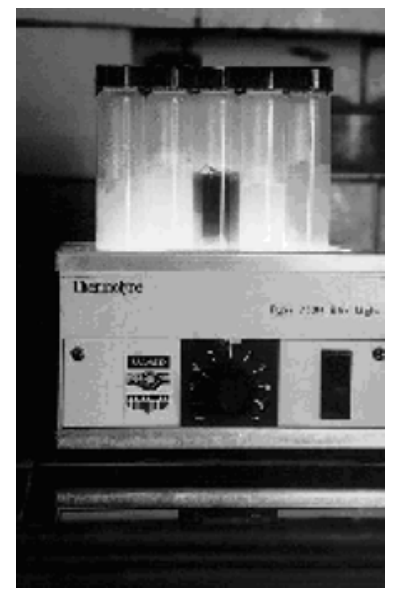

(b)

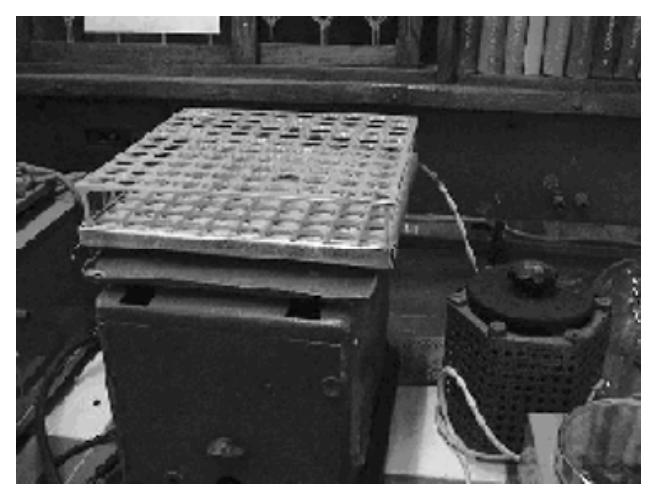

(c)

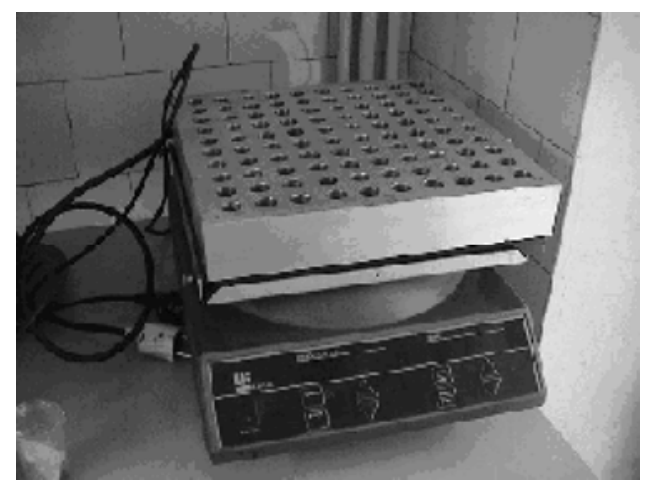

Fig. 4. Techniques for parallel heating of vials.

is equipped with a single reflux condenser (a hollow metallic module with 24 slots) which is mounted on cylindrical reactors. All vessels are covered on the top with a common metallic cap, through which one can add reagents or force an inert gas. The main "secret" of the cap, allowing evaporation of all the 24 vessels simultaneously will be discussed below.

\section{Parallel Boiling without Condensers}

High-temperature boiling of solutions has as long as two centuries been performed in the simplest autoclaves, viz. sealed tubes. It is this idea that is still widely exploited in combinatorial chemistry, the only thing is that the reaction vessel here is a thermal-glass tube or plain-bottomed vial with a tight screw cap (Fig. 3).

As a rule, the cap for a heated vial has a Teflon rubber seal. Such construction is quite strong and allows overheating above the boiling points of solvents (which is actually equivalent to using sealed ampules). After cooling, excess pressure is readily released by piercing the seals. In our experiments, vials with acetonitrile solutions $\left(\mathrm{mp} 82^{\circ} \mathrm{C}\right)$ tolerated many-hour heating at $100^{\circ} \mathrm{C}$. A few vials can be stirred with a usual magnetic stirrer without heating (Fig. 4a). For a large number of vials one can make a simple support, fix it on a shaker, and bottom-heat vials by means of an electric heating coil attached to a standard laboratory transformer (Fig. 4b, after D. Al'bov).

The safest approach is to heat hermetically sealed vials in the holes of temperature-controlled block heaters whose design (and well shapes) may vary. We successfully tried a J-Kem 310 block heater of a $10 \times 10$ format (Fig. 4c) with a timer and electronic controller of temperature and shaking intensity.

\section{Synthesis in Microwave Ovens}

Reactions in microwave (MW) ovens are much shorter and thus meet the main requirement of combinatorial chemistry, namely acceleration of synthesis. There are two ways to perform such reactions. The first is to perform a great number of reactions simultaneously. A rotating support (carousel) loaded with a great number of reaction vessels is placed into an MW oven (Figs. 5a-5c). The magnetic stirrer built-in into the bottom of the apparatus provides a uniform stirring in each vial. In the second technology, the MW oven contains only one port for one vessel (Fig. 5d), and reactions are performed sequentially by means of a robotic module: The vials are changed in a computercontrolled sequence. Technical solutions are constantly improved: In the considered CEM models, the temperature in the reference vessel is measured with an optical fiber sensor, and automatic shutdown of heating is applied when the pressure in vessels gets rising.

\section{Syntheses in Flow Microreactors}

Among the most recent technical innovations for synthesis acceleration we would like to mention technologies based on flow microreactors. One hardly imagines a usual reaction vessel without a powerful mechanical or magnetic stirrer, since effective stirring is an indispensable condition for successful reaction; in the above examples, the same role was played by 
(a)

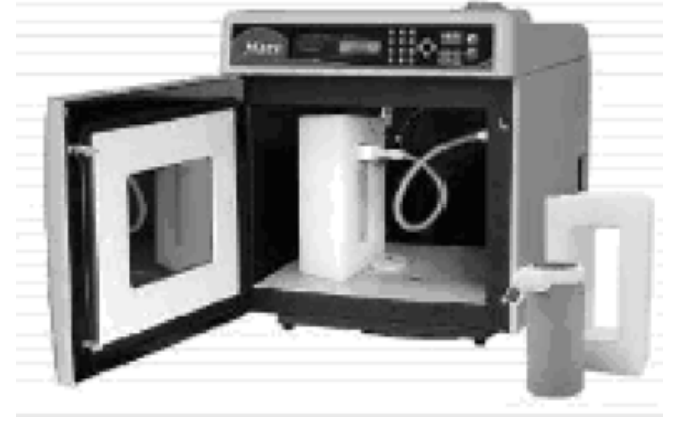

(b)

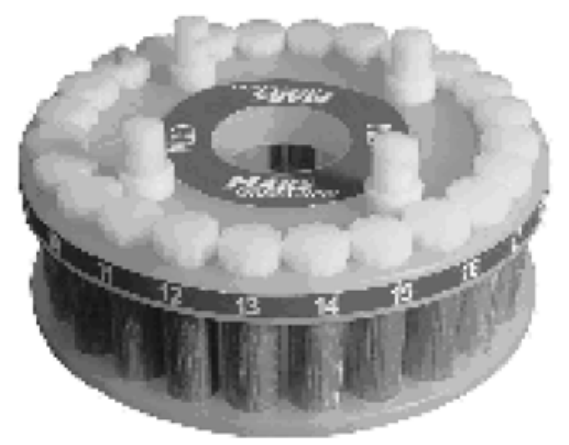

(d)
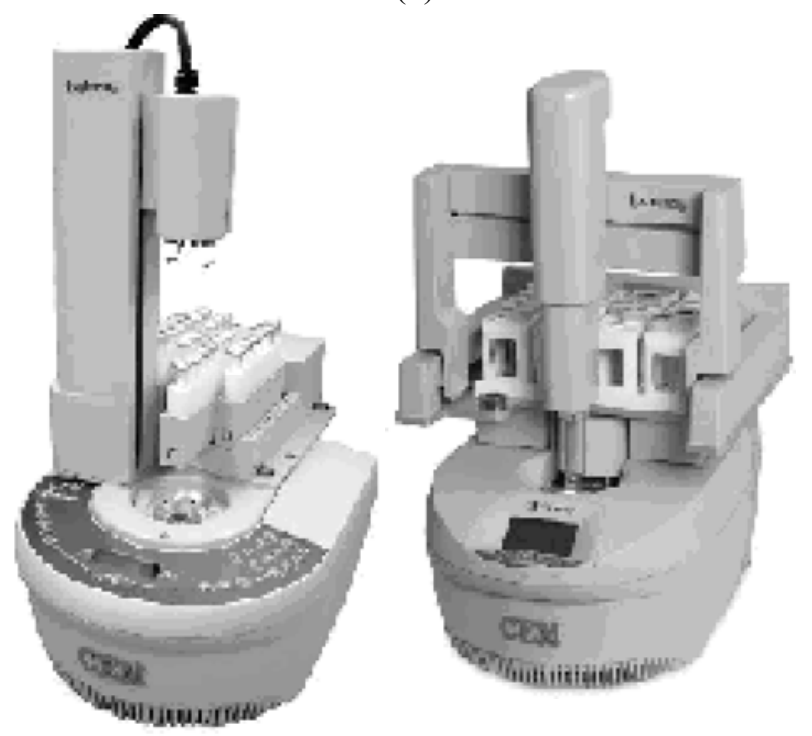

(c)

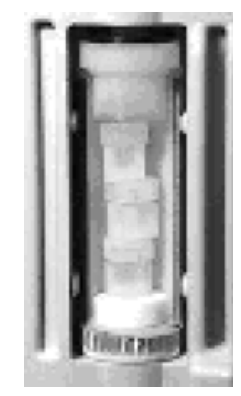

Fig. 5. Microwave ovens for combinatorial synthesis: (a) MW oven with a carousel for (b) glass and (c) plastic vessels in the MARS CEM model; (d) robotized attachments for fast sequential synthesis in the CEM Explorer model.

agitation in a shaker. In its essence, such stirring is turbulence, and its speed has a certain limit. This limit can be overcome by laminar diffusion; to induce this phenomemon, one has to make the volume where reagents meet each other close to zero (by performing reaction in a very thin capillary, Fig. 6a), and the rate of this meeting, to infinity (by applying a a very high pressure to inject reagents into this volume). Just this principle is realized in flow microreactors whose "heart" is formed by powerful pumps and "arteries," by extremely thin capillaries engraved (or chemically etched) inside quartz or plastic plates (Figs. 6b and 6c). Now the highest degree of reagent mixing has time to occur in the first millimeters of the capillary, taking fractions of a second. For the reaction to come to completion, the capillary should be long enough (a meter and longer). The plate with a capillary can be likened to a sealed vial (by "locking" the outgoing liquid by counter-pressure at the outlet); thus the flowing solvent can be overheated above its boiling point, thereby still more accelerating the process. In up-to-date microreactors, the reagent feeding and product collection system is robotized, and, therewith, for parallel synthesis several plates with capillaries are used (Fig. 6d). As a result, the synthesis is made fully automated and feasible for preparing both small libraries (on the micro scale) and tens and hundreds grams of a target product. Such "factories" (really substituting the whole plant floors) can be easily accommodated on a desktop. Figure 6e shows a fully robotized system Africa (Syrris) realizing this technology, which was purchased for our research. A great number of traditional reactions have already been optimized (see the specialized journal Lab on Chip): Now these reaction times are unimaginably short and the yields are sharply increased. 
(a)

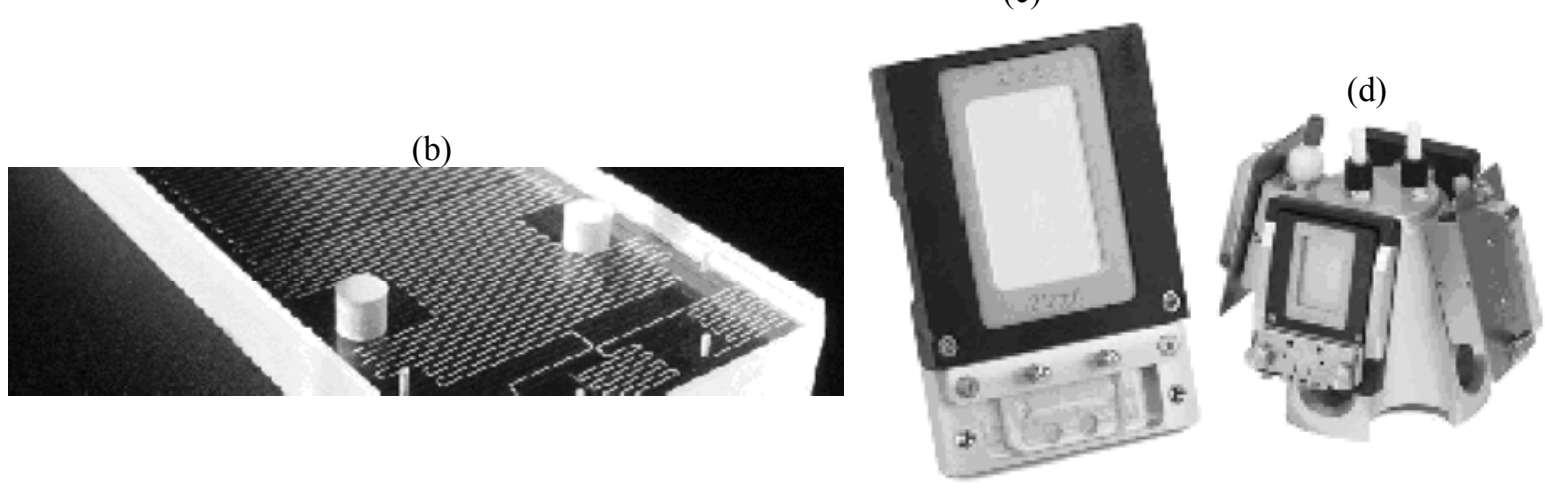

(e)

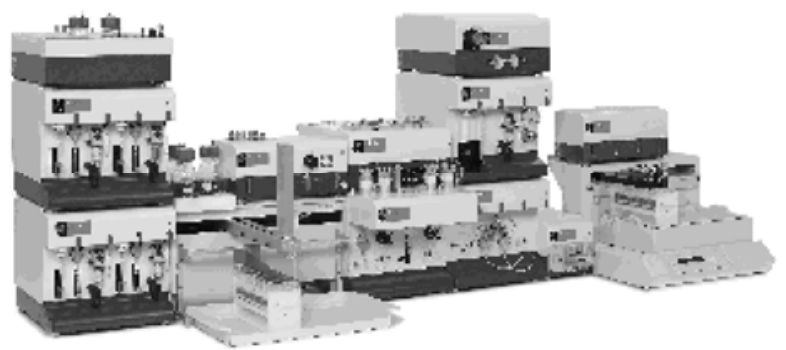

Fig. 6. Flow microreactors for fast synthesis: (a ) capillaries; (b, c) quartz or plastic plates; (d) block of several plates with capillaries; and (e) general view of a Syrius Africa microreactor.

\section{Separation of the Reaction Mixture}

Any parallel synthesis is inevitably followed by the stage of treatment of the reaction mixture, not infrequently quite a laborious process. Heterogeneous reactions with precipitate formation require filtration, and homogeneous reactions generally require extraction (either of the reaction mixtures in themselves or after their dilution with water). In phasetransfer reactions, too, phase separation is required.

\section{Parallel Extraction}

It is not very difficult to add water (or another solvent) simultaneously into several vessels; vigorous parallel agitation of emulsions in a shaker, too, is not quite a complicated problem. More difficult is to separate phases in parallel not resorting to classical separatory funnels. Several approaches are possible. One can act is a slightly unusual way and not to go beyond filtration or decantation. Since water freezes easier than other solvents used for extraction, the aqueous layer in the vial can be frozen out in a refrigerator and easily separated from the organic layer. Separatory funnels can be went without in a different way by equipping the reaction vial with a kind of "drain cock". To this end, a flexible plastic vial cap is pierced with two syringe needles (Fig. 6a). One needle is used to withdraw the required phase with a syringe, and the other needle (not immersed into the liquid) serves for pressure equalization.

Separation can also be accomplished using highcost automated workstations for parallel станций synthesis (Figs. 7b and 7c). A two-phase system is passed through a special sensor (B) sensitive to physical parameters at the phase interface (A), and the liquid flow is stopped in a due moment.

Emulsions can also be separated by means of a socalled "molecular filtering" (realized in the Africa autosynthesizer as a FLLEX attachment, Fig. 8a). Water is injected into the organic liquid flow (or vice versa), the resulting aqueous organic emulsion is forced under pressure through a membrane, and then again is separated into the components (Fig. 8b). The plastic membrane is quite small (Fig. 8f), and its pore size is so that only small water molecules can pass through (Fig. 8d).

\section{Parallel Filtration}

If precipitates are present in the reaction vials, it would be desirable to filter them in parallel, not 
(a)

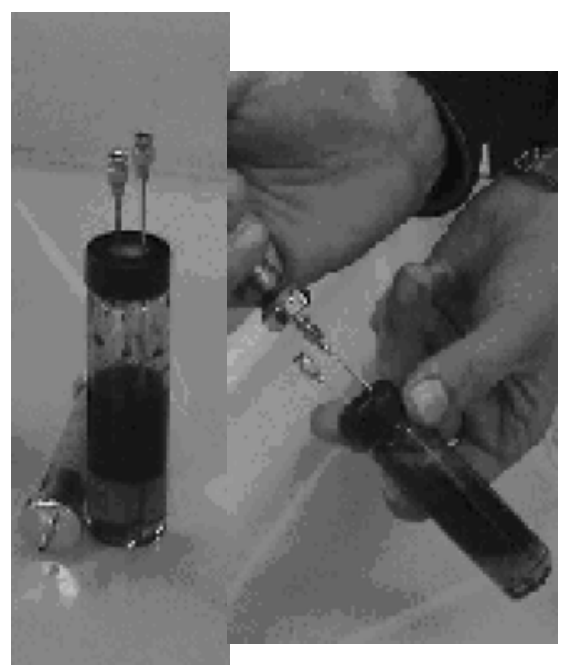

(b)

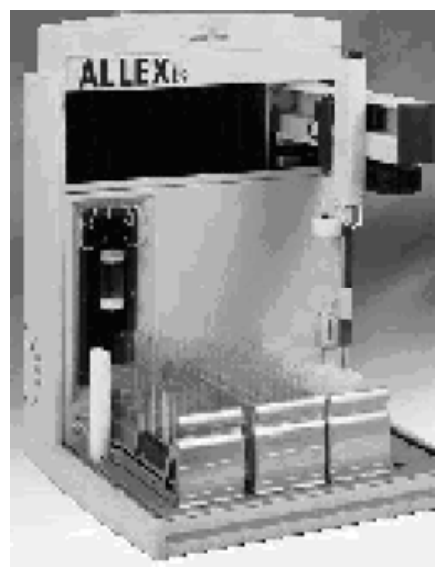

(c)

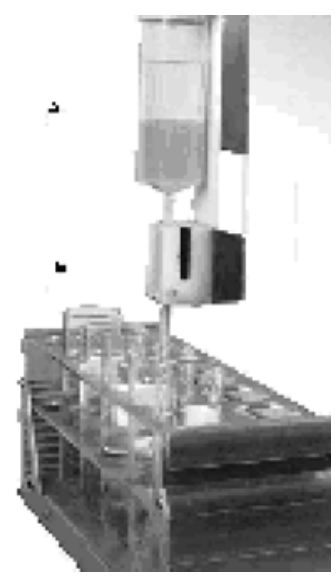

Fig. 7. Parallel extraction: (a) with a syringe; and (b, c) workstation for parallel synthesis.

transferring suspensions onto a filter and using a single pump. Reaction vessels combined with filters are in general use in solid-phase [2] and liquid-phase syntheses. If a reaction occurs at room temperature and

(a)

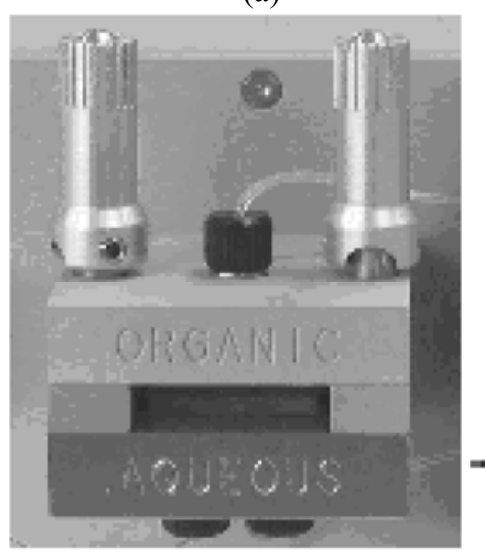

(c)

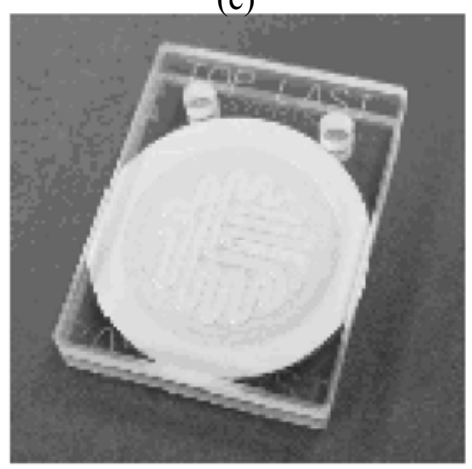

Fig. 8. Separation of a two-phase emulsion through a membrane in the FLLEX attachment in the Africa system. For denotations, see text. results in precipitate formation, it can be performed in a 96-well plastic plate (Figs. 9a-9c). Therewith, the billet bottom should be porous (by the principle vessel = filter). During reaction the billet is hermetized on the top and bottom with flexible seal caps. After reaction the caps are removed (Fig. 8a) and apply special facilities to vacuum filter 96 precipitates (Figs. $9 \mathrm{~b}$ and 9c) and to collect 96 filtrates.

For parallel filtration on a laboratory scale, "filtration lines" with a few filters are produced (Fig. 9d). Filters can be fixed in different ways (for example, with usual rubber stoppers), and the filtrate is collected in a common receiver.

The simple system for parallel filtration shown in Fig. 9d deserves special mentioning. Here each filtrate can be collected separately, by placing the support with receivers into a vacuum dessicator. This construction is also feasible for drying solutions by passing through a drier bed. The simplest device for simultaneous filtration of several solutions, with a standard vacuum dessicator and standard glass filters is shown in Fig. 9f, where the cap with several slots for filters is equipped with a single vacuum valve. As seen, all the above-mentioned techniques for filtering a great number of precipitates employ only one pump.

The CombiSyn (Fig. 2a) and SynCore (Fig. 2b) parallel synthesis systems realize a slightly different principle. In the first case (Fig. 9g), a plastic tube with a filter at the end is immersed into the vessel, and the solvent is pumped out in a vacuum. In the second case (Fig. 9h), filter tubes are pushed into each tube up to 
(a)

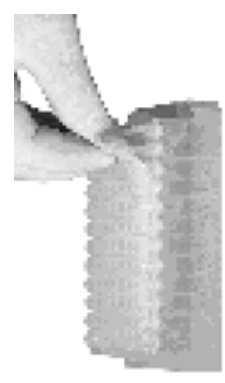

(e)

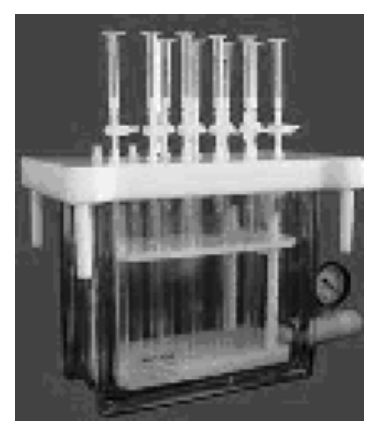

(b)

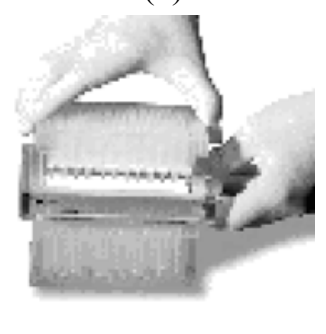

(c)

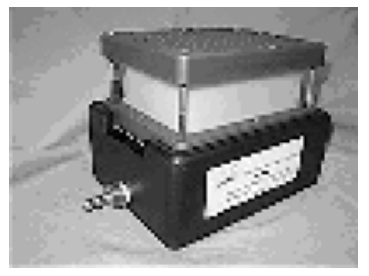

(g)

(d)

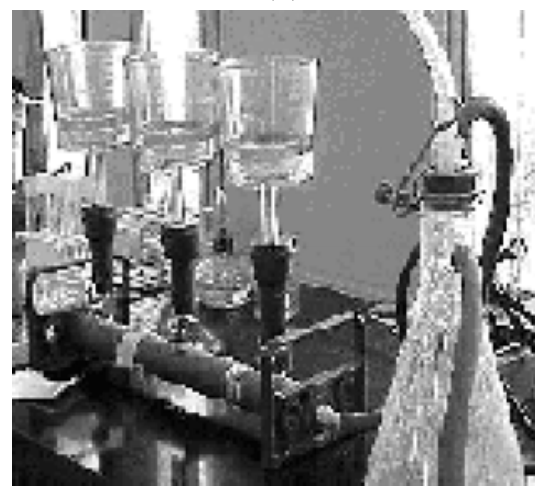

(h)

(f)

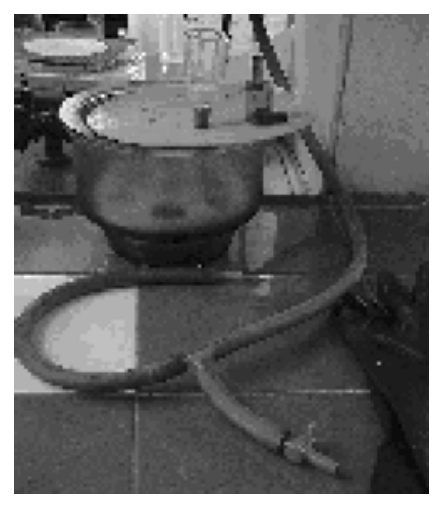

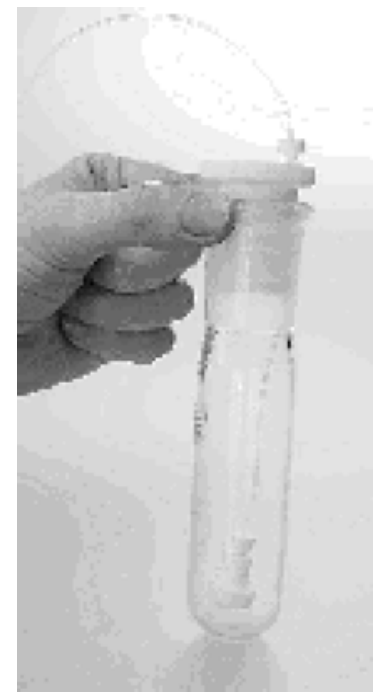

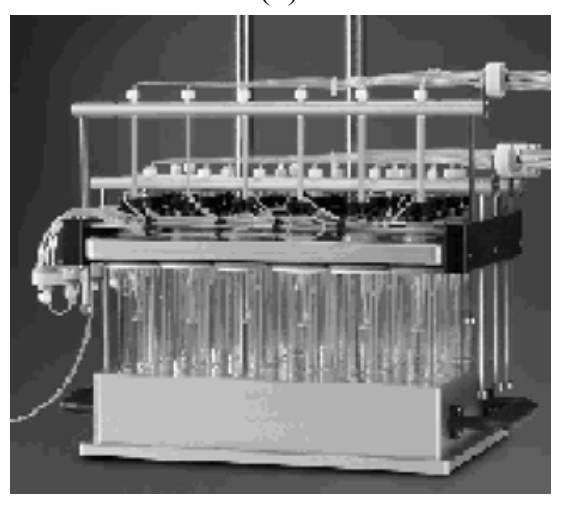

Fig. 9. Systems for parallel filtration. For denotations, see text.

the very bottom, a gas pressure is applied, due to which the solvent is forced through a system of prelabeled tubings into 24 receivers. We used just this technique in our training task [4].

\section{Parallel Centrifugation}

Parallel centrifuging is widely used for precipitate separation. To this end, attachments for great number of vessels and billets are used (Fig. 10), and the solvent is simply decanted. This technique is considered in detail in [5].

\section{Evaporation of Solutions}

Simultaneous evaporation of several solutions is likely to present the most serious problem of parallel LPS. In cases where special equipment is unavailable, there are three possible solutions: (1) sequential evaporation of a great number of samples on rotary evaporators; (2) long-term evaporation of open reaction vessels at ambient temperature and atmospheric pressure (for example, under a hood for light solvents); 3) use of a vacuum drying oven with a careful vacuum control to prevent solvent bumping.

The listed methods are unsuitable if one needs to a large series of solution in a high-boiling solvent (for example, DMSO which is frequently used in biological tests) or evaporate fairly large volumes $(10-100 \mathrm{ml})$ of extractant solvents. In such cases one need either a vacuum centrifuge or a system for parallel evaporation in a low vacuum.

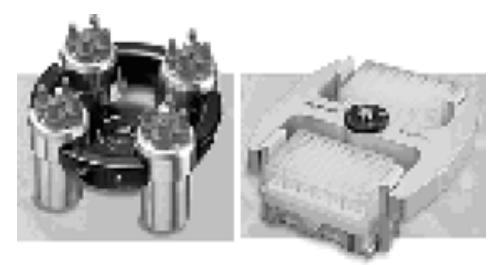

Fig. 10. Attachments for parallel centrifugation. 
(a)

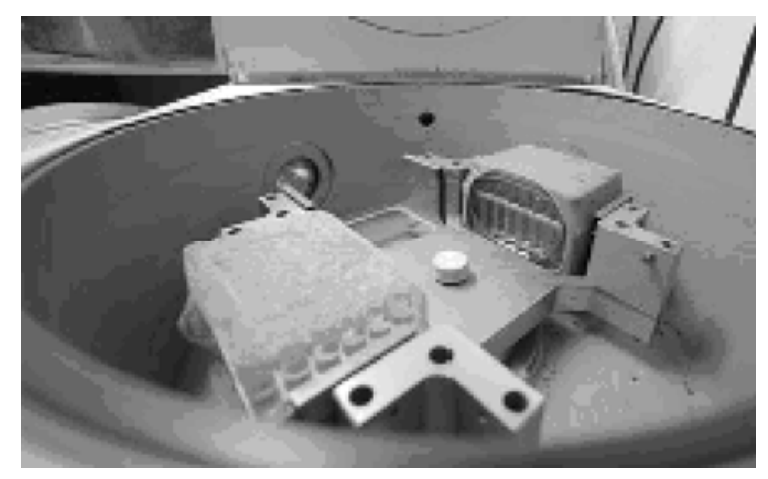

(b)

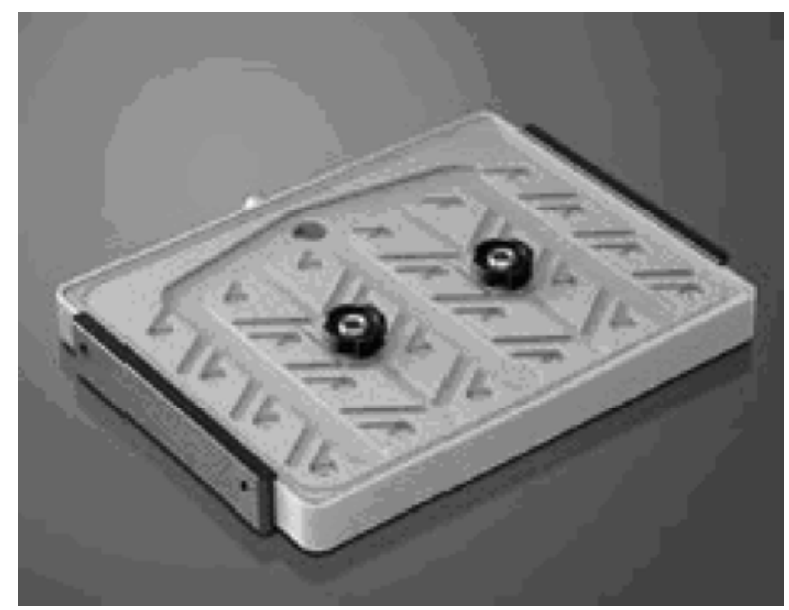

Fig. 11. Parallel evaporation by means of (a) vacuum centrifuge or (b) special hollow attachment to the Syncore apparatus, connected to a vacuum pump.

(a)

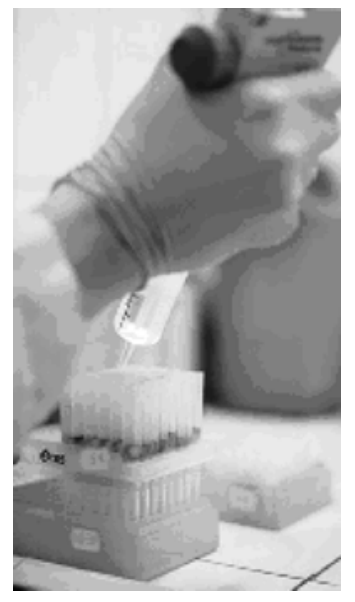

(b)

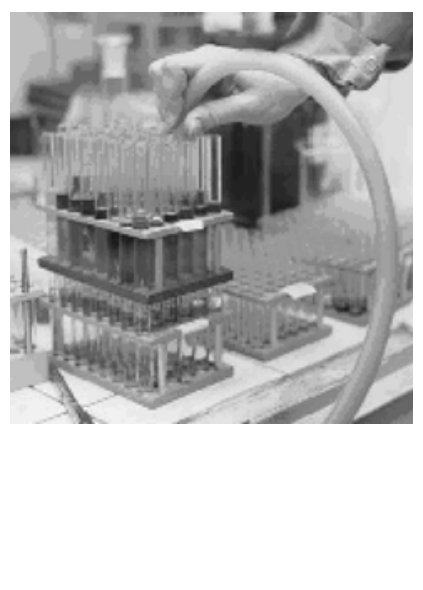

(c)

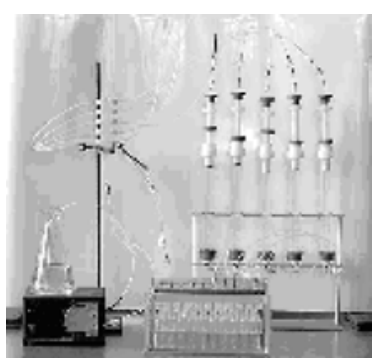

(d)

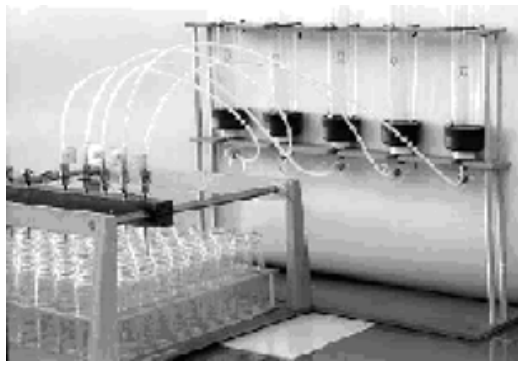

Fig. 12. Systems for parallel chromatography. For denotations, see text.

In a vacuum centrifuge (Fig. 11a) we can place individual vessels, billets with solutions, or plate holders with tubes. Bumping and cross-contamination are avoided due to a centrifugal force (sometimes billets are covered with a "net"). The evaporation rate can be increased by freezing out the distillate in the receiver. The SynCore apparatus (Figs. 2b and 11b) combines several techniques to prevent bumping and for preventing bumping and cross-contamination of solutions in neighboring vessels. Vacuum is set and maintained with an electronic controller. A hermetically sealed cap directs vapors through a sophisticated labyrinth of slots in the upper part of the cap, and, therefore, the splashed liquid returns back into the same vessel.

\section{Parallel Purification}

Purification of a great number of reaction mixtures is an unavoidable problem of LPS. To this end, the laboratories of major pharmaceutical companies make use of preparative liquid chromatographs with a programmed change of receiver at changing concentration of a substance passing through the column. Efficiency is provided due to full-time operation of several instruments. Unfortunately, the high cost of such equipment restricts its use at educational institutions. At the same time, there are a number of efficient low-cost techniques for parallel purification.

Solid-phase scavengers considerably facilitate purification of single-type reaction mixtures. The 
(a)

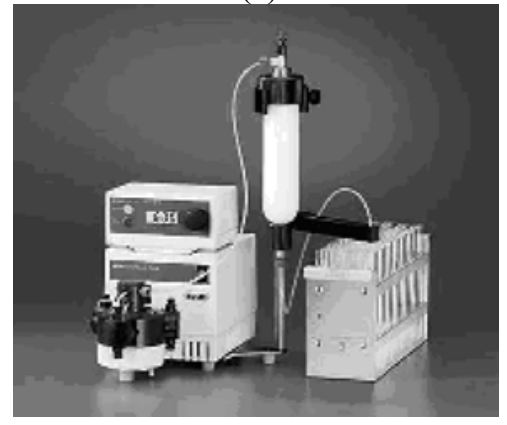

(b)

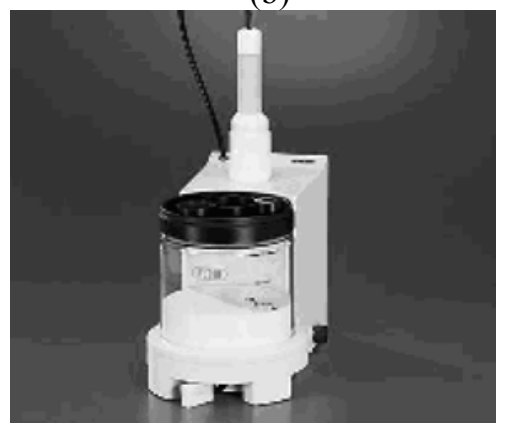

(c)

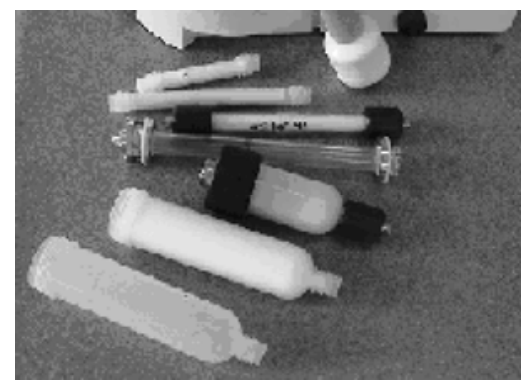

Fig. 13. Use of disposable columns in the Sepacore system. For denotations, see text.

(a)

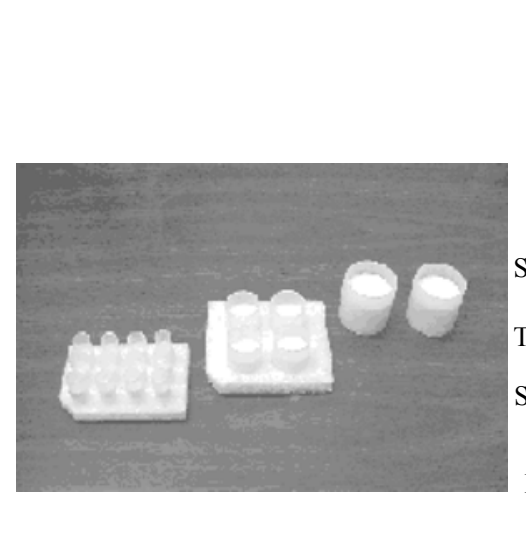

(b)

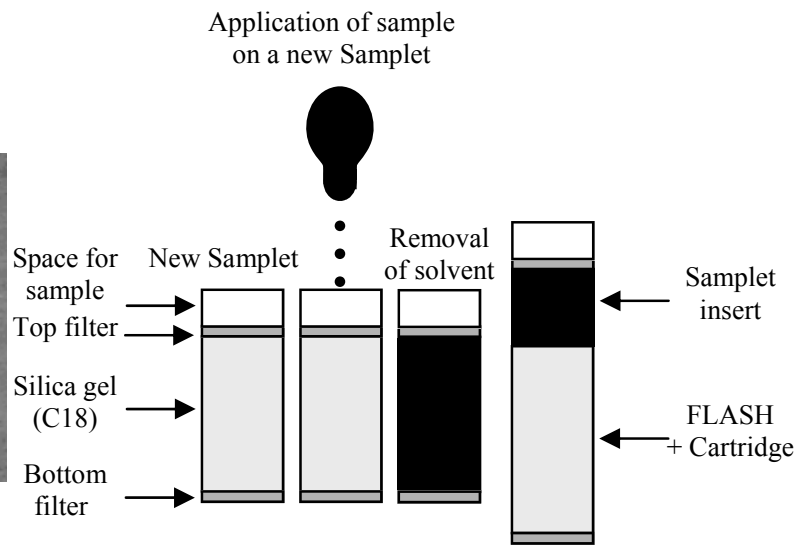

(c)

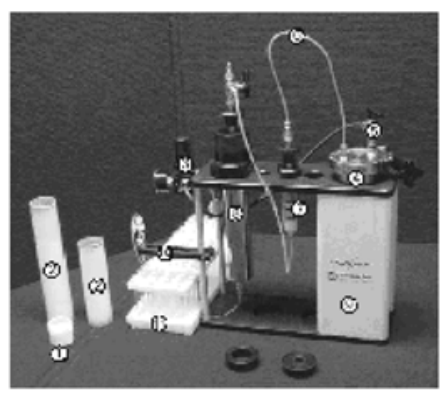

Fig. 14. Disposable samplet cartridges for preparative flash chromatography. For denotations, see text.

scavengers are polymer supports with an active functional group (sulfonic acid, tertiary amine, etc.) on the surface. If some reaction components (for example, amine, base, or acid) were taken in excess and are present in the final reaction mixture, they are reliably removed by scavengers. Technical details of operation with a simple scavenger are discussed in [4].

Parallel chromatography can be performed in different ways (Fig. 12). Low-cost billet filters $(4 \times 8$ или $8 \times 12$ ) which are convenient to use not only for parallel filtration (Fig. 8a-8f) but also for parallel chromatography (Fig. 12a). If chromatographic columns of a larger volume are needed, then glass column with solder-in filters are taken and compactly fixed on supports (Fig. 12b). Features of this technologies are discussed in [4] on a concrete example of reductive amination.

Chromatography in plastic or glass columns can be accelerated by either evacuation of the receiver or supplying an inert gas from a balloon onto the top of the column (Fig. 12b). In both cases, there is a threat to "overdry" the column and disturb uniformity of the separation process.

An interesting solution of this problem can be provided by a simple system for pumping of eluent through several columns simultaneously (Fig. 12c). One liquid supply pump and a simple system of distribution tubings for 5 columns are used. An automatic sample collector can be replaced by a convenient manual device (Fig. 12d).

Economical flash chromatography. At rigid purity requirements to library compounds, consecutive chromatographic purification of every individual sample, rather than parallel chromatography is required. How can we drive this process? We tried a Sepacore economical and efficient flash chromatography system. The system comprises a portable preparative chromatograph (Fig. 13a) with an isocratic pump, UV 
detector, manual sample collector, mini recorder, apparatus for superdense column packaging (Fig. 13b), and a set of cheap disposable plastic cartridges (Fig. 13c). Efficient column packaging is reached due to that a disposable tube column is evacuated from one end, immersed into a bed of silica or another sorbent, fluidized with nitrogen, and then tightly sealed with porous inserts by means of the device in Fig.13b. To transform a plastic tube into an efficient column takes a few minutes; such technique is readily applied for fast separation of a series of mixtures.

In certain cases, the sorbent in a column is inexpedient to change (especially, if reversed-phase or costly chiral sorbends are used), while multiple successive runs pose a risk of damage. The best solution in such situation is to use plastic disposable containers with sorbents (samplets) (Fig.14a), placed on the top of the column. Solutions with samples are applied on samplets in advance (Fig. 14b). During chromatography tarry products are retained on the insert, and after the separation is complete, the insert is replaced (Fig. 14c).

\section{CONCLUSIONS}

We would like to mention several trends in the development in techniques for liquid-phase combinatorial chemistry. First, equipment compactness and miniaturization (transfer from a flask to vial and even to capillary). Second, combining in a common space (vessel $=$ filter $=$ separatory funnel $=$ column $)$ traditionally incompatible processes (synthesis - workup - purification), which saves time. Third, sharp increase of the cost of the most efficient equipment, which is, to a certain extent, compensated for by the low cost of consumables. Therewith, glass is expelled by disposable plastic (billets, syringes, cartriges, samplets, etc.). Finally, the very idea of parallel processes is substituted by the idea of sequential processes which are, however, so fast (performed in an automated mode) that the total time consumption is reduced.

The LPS techniques are constantly fed by new ideas from SPS. We dwell on certain most illustrative examples [6]. One of the tendencies is extending use of solid-phase reagents and scavengers readily separable from reaction mixtures by simple filtration. Thus, solution pHs can now be controlled by acids and based immobilized on a support (i.e. not contaminating the solution with new mineral components), and oxidation can be performed by means of resin-supported oxidants (permanganates, priodates, etc. $[7,8]$ ). Further trend is to perform reactions in a homogeneous medium and transfer them, when necessary, to a heterogeneous phase. To this end, one can use, for example, polyethylene glycol, an excellent solvent which can be precipitated, when needed, by adding methanol. One more example is perfluorinated solvents which don't mix with conventional organic solvents. It was found that heterogeneous emulsions on the basis of perfluorinated components magically transform into homogeneous at slightly elevated temperatures. In this way, for example, one can "temporarily homogenize" a required reagent or catalyst and then remove it (by imparting to it a higher affinity to the perfluorinated phase). The use of molecules with fluorinated linkers for this purpose is considered in detail in [8]. As seen, the scope of traditional liquid-phase synthesis turns to be inexhaustable and is being more and more enriched with new scientific and technical solutions and inventions.

\section{REFERENCES}

1. Babaev, E.V. and Ermolat'ev, D.S., Ross. Khim. Zh. (Zh. Ross. Khim. O-va im. D.I. Mendeleeva), 2009, vol. 53 , no. 5 , p. 42.

2. Babaev, E.V., Ibid., p. 57.

3. Antonenko, V., Combinatorial Chemistry and Technology. Principles, Methods and Applications, Miertus, S. and Fassina, G., Eds., New York: Marcel Dekker, 1999, p. 205.

4. Ivanova, N.V., Tkach, N.V., Belykh, E.N., Dlinnykh, I.V., and Babaev, E.V., Ross. Khim. Zh. (Zh. Ross. Khim. O-va im. D.I. Mendeleeva), 2009, vol. 53, no. 5, p. 105.

5. Mironov, M.A. and Babaev, E.V., Ibid., p. 133.

6. Ferrito, R., De Magistris, E., Vissio, A., Haio, A., and Seneci, P., Combinatorial Chemistry and Technology. Principles, Methods and Applications, Miertus, S. and Fassina, G., Eds., New York: Marcel Dekker, 1999, p. 53.

7. Kironi, P., Al'vares, M., and Al'berisio, F., Ross. Khim. Zh. (Zh. Ross. Khim. O-va im. D.I. Mendeleeva), 2009, vol. 53, no. 5, p. 73.

8. Kurran, D., Ibid., p. 87. 